\title{
Paper \#77
}

\section{THE IMPACT OF SITTING CONFIGURATION ON THE HIP JOINT REACTION FORCE AND HIP FLEXION ANGLE}

Jan Van Houcke ${ }^{1}$, Ashwin Schouten ${ }^{2}$, Koen Vermeulen ${ }^{1}$, Gilles Van Acker ${ }^{1}$, Gunther Steenackers ${ }^{2}$, Christophe Pattyn ${ }^{1}$, Emmanuel Audenaert ${ }^{1}$

${ }^{1}$ University Hospital Ghent, Gent, Belgium, ${ }^{2}$ Antwerp, Belgium

FDA Status: Not Applicable

Summary: The kneeling chair configuration results in a $50 \%$ reduction of hip joint reaction force as well as a significantly lower hip flexion angle compared with a simple chair and especially a car seat.

Introduction: Deep seated sitting has been reported to produce pain in mechanical hip diseases, such as femoroacetabular impingement (FAI). Unlike lower back diseases, the effect of different sitting configurations on hip biomechanics has not yet been investigated. Since prolonged sitting is often an important part of the daily work and leisure activities of FAI patients, we examined the impact of 3 different sitting configurations on the hip joint reaction force and the hip flexion angle.

Methodology: Three musculoskeletal sitting models were built to simulate an average simple chair, car seat and kneeling chair configuration. A virtual average male Caucasian subject $(1.75 \mathrm{~m}, 74 \mathrm{~kg})$ was seated in the three distinct configurations and both static hip joint reaction force and hip flexion angle were measured.

Results: The observed median HJRF and hip flexion angle, respectively, was $124 \mathrm{~N}$ and $63^{\circ}$ for the simple chair, $132 \mathrm{~N}$ and $78^{\circ}$ for the car seat and $59 \mathrm{~N}$ and $51^{\circ}$ for the kneeling chair.

Discussion: A relative reduction of $50 \%$ in $\mathrm{HJRF}$ was observed in the kneeling chair configuration compared to the car seat and simple chair. More importantly, the hip flexion angle was significantly lower in the kneeling chair configuration and as such did not reach threshold values for femoroacetabular conflict. In conclusion, the kneeling chair appears to hold the greatest potential as ergonomic sitting configuration for the hip joint in case of FAI. 